\title{
Lateralized deficit of response inhibition in early-onset schizophrenia
}

\author{
MARK A. BELLGROVE ${ }^{1 *}$, CHRISTOPHER D. CHAMBERS ${ }^{1}$, ALASDAIR VANCE ${ }^{2}$, \\ NICOLE HALL ${ }^{2}$, MARY KARAMITSIOS ${ }^{3}$ AND JOHN L. BRADSHAW 3 \\ ${ }^{1}$ Cognitive Neuroscience Laboratory, School of Behavioural Science, University of Melbourne, Australia; \\ ${ }^{2}$ Academic Child Psychiatry Unit, Department of Paediatrics, University of Melbourne, The Royal Children's \\ Hospital and Murdoch Children's Research Institute, Australia $;{ }^{3}$ Department of Psychology,
}

Monash University, Australia

\begin{abstract}
Background. The ability to inhibit inappropriate or unwanted actions is a key element of executive control. The existence of executive function deficits in schizophrenia is consistent with frontal lobe theories of the disorder. Relatively few studies have examined response inhibition in schizophrenia, and none in adolescent patients with early-onset schizophrenia (EOS).
\end{abstract}

Methods. Twenty-one adolescents with the onset of clinically impairing psychosis before 19 years of age and 16 matched controls performed a stop-signal task to assess response inhibition. The patients with EOS were categorized as paranoid $(n=10)$ and undifferentiated subtypes $(n=11)$. The undifferentiated group had higher levels of negative symptomatology. Stop-signal reaction time (SSRT) and go-signal reaction time (Go-RT) were analysed with respect to hand of response.

Results. The undifferentiated early-onset patients had significantly longer SSRTs, indicative of poor response inhibition, for the left hand compared to the paranoid early-onset patients and control participants. No differences existed for inhibitory control with the right hand. The three groups did not differ in Go-RT.

Conclusions. Our results indicate a specific lateralized impairment of response inhibition in patients with undifferentiated, but not paranoid, EOS. These findings are consistent with reports of immature frontostriatal networks in EOS and implicate areas such as the pre-motor cortex and supplementary motor area (SMA) that are thought to play a role in both voluntary initiation and inhibition of movement.

\section{INTRODUCTION}

Executive dysfunction is a prominent feature of schizophrenia (Kolb \& Wishaw, 1983; Berman et al. 1986; Pantelis et al. 1997). The existence of executive deficits in schizophrenia is consistent with frontal lobe theories advanced by Kraepelin (1913) and Bleuler (1911), and confirmed by modern neuroimaging techniques (Berman et al. 1986; Buchsbaum et al. 1992;

\footnotetext{
* Address for correspondence: Dr Mark A. Bellgrove, Cognitive Neuroscience Laboratory, School of Behavioural Science, The University of Melbourne, Parkville 3010, Victoria, Australia. (Email: bema@unimelb.edu.au)
}

Hill et al. 2004; Kumra et al. 2004). Executive function comprises distinct aspects of response inhibition, working memory, set-shifting and interference control (Pennington \& Ozonoff, 1996; Miyake et al. 2000). Although deficits in working memory, set-shifting and interference control are well established in schizophrenia, relatively few studies have examined response inhibition (Kiehl et al. 2000; Weisbrod et al. 2000; Rubia et al. 2001 a; Badcock et al. 2002; Ford et al. 2004). It remains controversial whether cognitive deficits are more pronounced in those patients with a relatively earlier onset of schizophrenic illness (Kravariti et al. 2003 b; 
Pantelis et al. 2003). The objective of this paper was therefore to examine response inhibition in patients with early-onset schizophrenia (EOS).

Although the cognitive mechanisms underlying response inhibition have been studied within cognitive psychology for many decades (Logan \& Cowan, 1984; Logan, 1994; Logan et al. 1997), it is only relatively recently that the neural basis of inhibitory control in the human brain has been investigated. Human lesion and neuroimaging studies support the view that response inhibition is achieved throughout a neural network including the inferior (IFG) and middle frontal gyri (MFG), supplementary motor area (SMA) and the inferior parietal lobule (Garavan et al. 1999; Rubia et al. 2001 b; Aron et al. 2003). Activation foci may also be seen in midline regions, such as the anterior cingulate (Rubia et al. 2001 b), although such activations may reflect ancillary processes such as monitoring response conflict (Carter et al. 1998) or error detection (Garavan et al. 2003), rather than response inhibition per se. Human lesion (Aron et al. 2003), split brain (Funnell et al. 2004), transcranial magnetic stimulation (TMS) (Chambers et al. in press) and functional imaging studies all suggest a dominant role of the right hemisphere, particularly the IFG, in response inhibition (Konishi et al. 1998; Garavan et al. 1999; Ford et al. 2004; Kelly et al. 2004).

Relatively few studies have examined response inhibition in schizophrenia (Kiehl et al. 2000; Weisbrod et al. 2000; Rubia et al. 2001a; Badcock et al. 2002; Ford et al. 2004) and results are conflicting. The majority of these studies have used classic go/no-go paradigms in which a response to an infrequent target stimulus (the no-go stimulus) must be withheld. Typically, prepotency to the go-stimulus is established by presenting many more go stimuli than no-go stimuli. Of those studies using go/no-go paradigms with schizophrenia and control groups, two have reported no behavioural differences (Fallgatter \& Muller, 2001; Rubia et al. 2001a), two have reported impaired response inhibition in schizophrenia (Kiehl et al. 2000; Weisbrod et al. 2000), and one has reported superior inhibitory performance in patients with schizophrenia (Ford et al. 2004).

Electrophysiological studies of response inhibition in schizophrenia are also inconclusive, with the N2 component, for example, reduced in some studies (Kiehl et al. 2000) but not in others (Weisbrod et al. 2000; Ford et al. 2004). Neuroimaging studies find intact inhibitory performance despite reduced activation in the left anterior cingulate and rostral dorsolateral prefrontal cortex (Rubia et al. 2001 a). Alternatively, decreased activation in right frontal and parietal regions has been reported (Ford et al. 2004). Inconsistencies may have arisen for several reasons. First, the go/no-go paradigm is susceptible to changes in response criteria over time, or between participants. Failure to establish the prepotent go-response may therefore reflect a strategic performance difference and a cautious response style (compared with Ford et al. 2004). Second, differences in the clinical samples also make interpretation difficult. For example, Rubia et al. (2001 a) recruited patients with a recent illness onset, whereas others recruited patients with chronic illnesses (Weisbrod et al. 2000; Fallgatter \& Muller, 2001; Ford et al. 2004). Generalized slowness in the latter group could confound performance on go/no-go tasks.

\section{The stop-signal paradigm}

An alternative paradigm for the investigation of response inhibition is the stop-signal paradigm. In this task, an established pattern of responding to a go-signal must be inhibited upon presentation of a countermanding stop-signal Typically the go-task involves a choicereaction-time (CRT) decision, requiring, for example, left and right button presses upon presentation of the letters ' $\mathrm{X}$ ' and ' $\mathrm{O}$ ' respectively (Logan et al. 1997). The stop-task, typically occurring in $25 \%$ of trials, signals participants to withhold their response to the go-signal. Instructions typically emphasize speed of responding, and successful inhibition can therefore be conceptualized as a race between competing go- and stop-processes.

The main dependent variable in the stopsignal paradigm is the stop-signal reaction time (SSRT). The SSRT is measured by introducing a stop-signal delay (SSD) between the presentation of the go- and stop-signals, so that the latency of the stop-process can be measured indirectly. SSDs are either selected randomly from a fixed array (method of constants; Logan \& Cowan, 1984) or varied dynamically 
contingent upon the response of the participant (method of limits; Osman et al. 1986; Kornblum et al. 1990; Logan et al. 1997). The SSD at which the participant can successfully inhibit $50 \%$ of the stop-trials represents the amount of processing time required to tie the race between the go- and stop-processes (Osman et al. 1986; Kornblum et al. 1990). As this delay represents the average point in time of the completion of the stop-process, it can be used to estimate the SSRT. The SSRT can be derived as the difference between the mean go-signal RT (Go-RT) and the delay at which the participant inhibits correctly on $50 \%$ of stop-signal trials (Osman et al. 1986, 1990; Logan, 1994; Logan et al. 1997).

Badcock et al. (2002) used the stop-signal paradigm in patients with schizophrenia, a psychosis comparison group and healthy controls. To control for shifts in response criteria, they adopted a method of constants and set six SSDs relative to each subject's own Go-RT. Although the groups did not differ in terms of their SSRT, the relationship between SSD and inhibitory performance was significantly flatter in the schizophrenia group compared to the psychosis group or the healthy controls. Badcock and colleagues argued that in schizophrenia there is an inability to trigger the inhibitory act, as indicated by a flatter inhibition function, but that once this act was triggered, the speed of inhibition was comparable to that of the control groups.

The present study sought to extend the previous literature in two important ways. First, we recruited a sample of adolescents with earlyonset schizophrenia (EOS), that is an onset of schizophrenic illness prior to 19 years of age (Bellgrove et al. 2003, 2004). Although earlyand adult-onset forms of the disorder may share the same pathophysiological substrates (Hollis, 2000; Nicolson et al. 2000), EOS may be characterized by a relative neurodevelopmental delay. Specifically, adolescents with EOS are developmentally delayed prior to the onset of psychosis (Hollis, 1995) and may show a persistence of neurological soft signs (Karp et al. 2001). Using diffusion tensor imaging, Kumra et al. (2004) reported reduced white matter integrity in the frontal lobes, including the IFG, of adolescents with EOS. Dysfunction within the IFG has been suggested as the pathophysiological substrate of negative symptoms in schizophrenia (Wolkin et al. 2003). These lines of evidence suggest that immaturity of frontostriatal neural networks (James et al. 2004), including the IFG, might confer vulnerability to an early onset of schizophrenia. We assayed the functional integrity of inhibitory networks in EOS using a stop-signal task. We predicted that inhibitory deficits would be most pronounced in those patients with higher negative symptoms.

Second, we applied an adaptive psychophysical staircase method to the problem of estimating the $50 \%$ correct inhibition threshold. Adaptive staircase methods are highly applicable to the estimation of the inhibition threshold because they place most observations close to threshold (Levitt, 1971). Furthermore, most previous studies have examined inhibitory performance within the stop-signal paradigm without regard to response hand (Logan \& Cowan, 1984; Osman et al. 1986, 1990; Logan et al. 1997). Arguments have been made, however, that motor areas such as the SMA may play a role in both initiating and inhibiting voluntary action (Badcock et al. 2002). As each laterality is controlled by motor areas in the contralateral hemisphere, hand of response may be an important factor to consider in response inhibition. This may be particularly germane to schizophrenia, where anomalies of motoric areas have been observed (Dreher et al. 1999; Payoux et al. 2004; Rogowska et al. 2004). The present study therefore used for the first time two independent, interleaved staircases to assess inhibitory control as a function of response hand in adolescents with EOS.

\section{METHOD}

\section{Participants}

Adolescents with early-onset adolescent schizophrenia and healthy controls participated in this study. Written informed consent was provided by the participant or the parent of the participant.

Twenty-one adolescent patients (12 male) with a diagnosis of EOS (DSM-IV; APA, 1994) were recruited from in- and out-patient adolescent units in metropolitan Melbourne, Australia. Patients were defined as being of adolescent early-onset if clinically impairing 
psychotic symptoms first appeared after the age of 12 but before 19. Patients were diagnosed categorically through a semi-structured clinical interview using DSM-IV criteria (K-SADS-PL; Kaufman et al. 1999) and dimensionally through the Positive and Negative Symptom Scale (PANSS; Kay et al. 1987).

The EOS group had a mean PANSS positive scale score of 21 (s.D. $=5 \cdot 6)$ (60th percentile), a mean negative scale score of 26 (s.D. $=5.9)(75$ th percentile), a mean general psychopathological scale score of 53 (S.D. $=11 \cdot 5)$ (90th percentile), and a mean total symptom score of 100 (s.D. $=$ $21 \cdot 5)$. Patients were categorized according to DSM-IV subtype: 10 of these participants were diagnosed as having paranoid EOS while 11 were diagnosed as having undifferentiated EOS. The undifferentiated group had higher scores on the negative scale of the PANSS than the paranoid group $[t(18)=2 \cdot 1, p=0 \cdot 05]$ but did not differ on the other dimensions.

The paranoid EOS group had a mean age of 16.9 years (S.D. $=1.7)$ and the undifferentiated EOS group had a mean age of 13.9 years (s.D. $=2 \cdot 5)$. Participants were administered the age-appropriate Wechsler Standardized Achievement Test; the paranoid and undifferentiated groups exhibited IQs within the average range (paranoid EOS: mean $\mathrm{IQ}=96$, s.D. $=$ 12.8; undifferentiated EOS: mean $\mathrm{IQ}=100$, S.D. $=13 \cdot 7)$. Fifteen of the patients were unmedicated and the remaining six were taking atypical neuroleptics at the time of testing, with equal numbers of medication naive and medicated participants in the paranoid and undifferentiated EOS groups. All of the paranoid EOS patients were right-handed as determined by a handedness questionnaire (Patterson \& Bradshaw, 1975), while 10 of the 11 undifferentiated EOS patients were right-handed. Participants had normal or corrected-to-normal vision and no colour blindness.

A group of 16 control participants (11 male, 13 right-handed) was recruited from the general community and from Rossbourne Secondary College, Hawthorn, Melbourne. These subjects were free of neurological or psychiatric disturbance, had a mean age of 15.9 years $($ S.D. $=1 \cdot 7$ ) and a mean IQ of $99 \cdot 6$ (s.D. $=10 \cdot 3)$. The three participant groups (paranoid EOS versus undifferentiated EOS versus controls) did not differ in terms of IQ $[F(2,34)=0 \cdot 38, p>0 \cdot 05]$ but did differ in age $[F(2,34)=6 \cdot 52, p<0 \cdot 05]$, with the undifferentiated EOS patients being younger than both the paranoid EOS patients and controls.

\section{Apparatus and procedures}

Participants sat in front of an active-screen Toshiba Satellite Pro 4300 portable computer, with the screen centred on their sagittal midline. Participants rested the index fingers of their left and right hands on two response buttons, fixed within a response box. Responses were executed by pressing one of the buttons as quickly and as accurately as possible. Reaction times (RTs) were recorded to the nearest millisecond (ms).

Participants performed a stop-signal task, similar in nature to that described by other authors (Logan et al. 1997). The experiment used a simple one-up one-down adaptive staircase method to estimate the SSD for the $50 \%$ inhibition threshold. This procedure has the advantage of placing most observations near the $50 \%$ point, thereby increasing the power and efficiency of the experiment (Levitt, 1971). The increments by which the onset (i.e. SSDs) of the stop-signal are either increased or decreased are called steps.

The step-size of the SSDs was tied to the standard deviation (S.D.) of the participant's RT distribution for Go-responses defined in a pre-experimental phase (see below). Calibrating the step-size to the participant's Go-RT distribution has several advantages. First, the pattern of step-size adjustments is subjectively equivalent for all subjects, which should equalize the difficulty of the task both within and between the patient and control groups. This manipulation should minimize error variance and maximize statistical power. Second, this method increases the efficiency of the adaptive staircase by using a final step-size tailored for that participant's psychometric function (i.e. it will not be too small and therefore inefficient, or too large and therefore inaccurate). Finally, our approach aims to homogenize criterion shifts (slowing of Go-RT to inhibit upon more occasions) that could unduly influence the value of the $50 \%$ correct inhibition point across participants.

The following section describes the procedures used for all participants leading to the estimation of the $50 \%$ correct inhibition point and calculation of the SSRT. The experiment 
consisted of three phases: a practice phase, a pre-experimental phase, and an experimental phase.

\section{Practice phase}

Participants completed 20 practice trials. The Go-stimuli were an uppercase ' $\mathrm{X}$ ' or ' $\mathrm{O}$ ', yellow on a black background, presented centrally for $1000 \mathrm{~ms}$ or until a response was executed. The ' $\mathrm{X}$ ' was $27 \mathrm{~mm}$ in height and $25 \mathrm{~mm}$ in width (visual angles $3.09^{\circ}$ and $2.86^{\circ}$ respectively) and the ' $\mathrm{O}$ ' was $29 \mathrm{~mm}$ in height and $28 \mathrm{~mm}$ in width (visual angles $3 \cdot 32^{\circ}$ and $3 \cdot 21^{\circ}$ respectively). The ' $\mathrm{X}$ ' was mapped to a left response and the ' $\mathrm{O}$ ' to a right response. A $500 \mathrm{~ms}$ fixation point, followed by a variable screen blank (200-1000 ms), preceded the presentation of the Go-stimulus. Equal numbers of the Go-stimuli were presented in a random order (i.e. $10 \mathrm{X}$ 's and $10 \mathrm{O}$ 's), with the stop-signal presented randomly on $25 \%$ of occasions. The stop-signal was a $100 \mathrm{~ms}, 1000 \mathrm{~Hz}$ tone presented through an external speaker attached to the response apparatus. SSD was varied randomly between 0 and $400 \mathrm{~ms}$. Once initiated, trials proceeded continuously. Participants were instructed to respond to the Go-stimuli as quickly as possible and to inhibit responding when they heard the tone. The requirement for speed of response was reinforced in the practice session by computer-generated feedback (accuracy and RT) provided between trials, which encouraged the participant to 'go faster'.

\section{Pre-experimental phase}

The pre-experimental phase comprised 200 trials presented in four blocks of 50 , and was designed to establish the participant's Go-RT distribution and yield the S.D. of this distribution for setting the staircase step-size in the experimental phase. This phase proceeded in the same manner as the practice phase, with the exception that the feedback regarding the correct response was withdrawn. Stop-trials were presented randomly on $25 \%$ of occasions with the SSD varied randomly between 0 and $400 \mathrm{~ms}$. Speed of responses was again emphasized by the experimenter.

\section{Experimental phase}

In the experimental phase, the s.D. of each participant's Go-RT distribution from the pre-

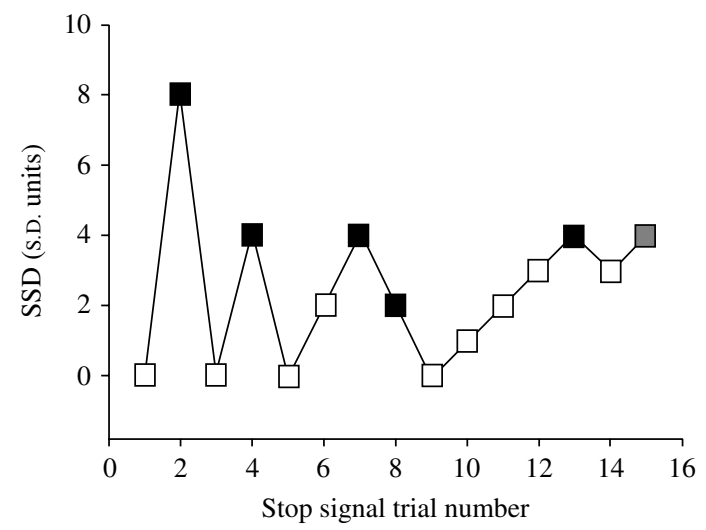

FIG. 1. A hypothetical staircase track adopting the $50 \%$ adaptive method. Black squares represent a correctly inhibited stop-trial, white squares represent a failed inhibition on a stop-trial, and the grey square represents the threshold value (s.D. units) or $50 \%$ correct inhibition point. Note that the first stop-signal is coincident with the go-signal $(\mathrm{SSD}=0)$. The second stop-signal, assuming correct inhibition on the first, was presented at a delay of $8 \times$ s.D. Thereafter the staircase followed a one-up one-down staircase.

experimental phase was used to set the step-size for an adaptive staircase to estimate the $50 \%$ inhibition threshold.

\section{Setting the step sizes}

From an initially tested SSD of 0 , the step-size (ms) was increased to a large multiple of the participant's Go-RT S.D. $(8 \times$ S.D. $)$ (see Fig. 1). Correct inhibition at this maximum SSD was permitted only five times before the session was terminated. This procedure safeguarded against extreme criterion shifts that could occur across the testing session. Following a failed inhibition, the next stop-trial was presented at the most recently correctly inhibited SSD. If the response was correctly inhibited twice running, then the step-size was halved (in S.D. units) and the SSD increased by the corresponding step-size (ms). Step-size (in S.D. units) was therefore halved after two correct inhibitions at the same SSD. Trials continued until a failed inhibition occurred at the minimum step-size. The minimum step-size was determined by halving the s.D. eight times. The $50 \%$ correct inhibition point was then taken as the SSD (ms) at which the last correct inhibition occurred.

\section{Independent adaptive staircases for left and right responses}

As it was of interest whether response inhibition varied as a function of response hand, two 
Table 1. Definitions for each of the four possible response types within the stop-signal paradigm

\begin{tabular}{ll}
\hline \hline Error type & \multicolumn{1}{c}{ Definition } \\
\hline Incorrect responses & $\begin{array}{l}\text { Incorrect response on a Go-trial } \\
\text { Incorrect failed inhibition }\end{array}$ \\
Missed trials & inhibition on a Stop-trial \\
False alarm & Missed detection on a Go-trial \\
& Correct response on a Go-trial but \\
sooner than $100 \mathrm{~ms}$
\end{tabular}

adaptive staircases were administered independently for left and right responses. This was achieved by setting step-sizes separately for each of the left and right responses, based upon the S.D. of the Go-RT distributions established for each hand in the pre-experimental phase. When the $50 \%$ correct inhibition point for one hand was reached prior to that of the other, all remaining stop-trials for the completed laterality were presented with an SSD equal to this obtained value, until the inhibition threshold of the other hand was reached.

The experimental phase proceeded in blocks of 50 trials until the participants had reached their $50 \%$ correct inhibition point for both hands. During this phase, speed of response was emphasized, and participants received a 'Go Faster' prompt if their Go-RT for a response slowed to greater than the longest correct Go-RT of the respective hand from the pre-experimental phase. Preliminary analysis indicated that the Go-RT distributions for each of the two groups did not differ statistically between the pre-experimental and experimental phases.

\section{Treatment of errors and outliers}

Incorrect responses (e.g. pressing the wrong button on a Go-trial) did not contribute to the Go-RT distributions in either the preexperimental or experimental phases. Step-size adjustments were implemented only after a correct or failed inhibition. During the experimental phase, all instances of errors were recorded for subsequent analysis. Four different response types were possible (Table 1). In both the pre-experimental and experimental phases, correct Go-RT values \pm three standard deviations were excluded from the analysis.

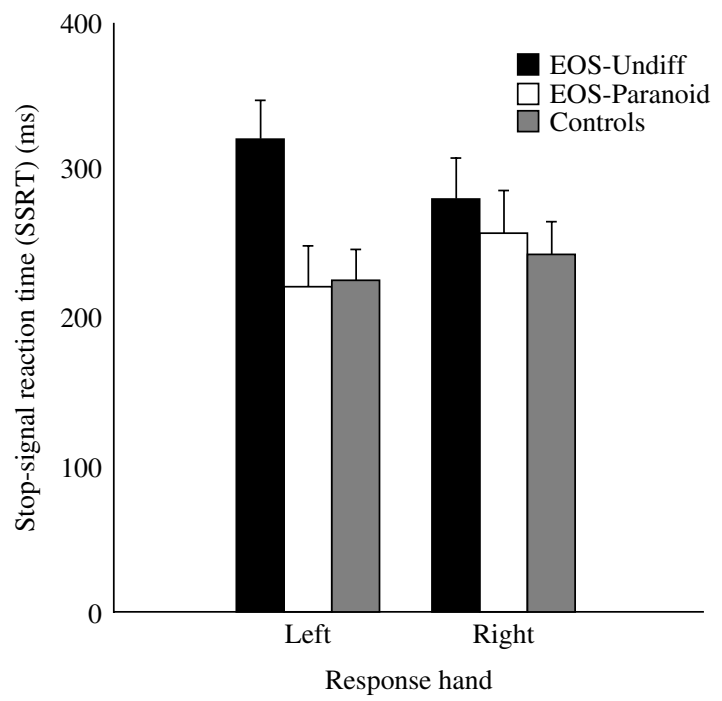

FIG. 2. Stop-signal reaction time (SSRT) (ms) for left and right response hands for each of the early-onset schizophrenia (EOS) and control groups. Undiff, Undifferentiated subtype.

\section{RESULTS \\ Analysis of SSRT}

SSRT was calculated by subtracting the SSD corresponding to the $50 \%$ inhibition threshold from the mean Go-RT (Logan et al. 1997). This calculation was performed separately for each hand. As mean age differed across groups, it was important to determine the relationship between age and SSRT, as a function of hand of response. Partial correlations between age, lefthand SSRT and right-hand SSRT (controlling for group) revealed no significant correlations (all $r$ 's $<0 \cdot 13$ ). Accordingly, we conducted a group (paranoid EOS versus undifferentiated EOS versus controls) by response hand (left versus right) mixed-model analysis of variance (ANOVA) on the SSRTs. There were no main effects of response hand $[F(1,34)=0 \cdot 25, p=$ $0 \cdot 62]$ or group $[F(2,34)=2 \cdot 43, p=0 \cdot 10]$. There was a significant interaction between group and response hand $[F(2,34)=3 \cdot 74, p=0.03$, $\left.\eta^{2}=0 \cdot 18\right] \quad$ (Fig. 2). Bonferroni comparisons revealed that this interaction was driven by the significantly longer SSRT of the undifferentiated EOS group with the left hand, relative to that of both the control $(p=0.03)$ and paranoid EOS $(p<0.04)$ groups. There was no significant difference between the control and 
Table 2. Mean percentage error rates and standard deviations for the early-onset patient groups and the control group, for each of incorrect, incorrect failed inhibitions, missed trials, and false alarm error types

\begin{tabular}{|c|c|c|c|c|}
\hline & \multicolumn{4}{|c|}{ Error type } \\
\hline & $\begin{array}{l}\text { Incorrect } \\
\text { responses }\end{array}$ & $\begin{array}{l}\text { Incorrect } \\
\text { failed } \\
\text { inhibitions }\end{array}$ & $\begin{array}{l}\text { Missed } \\
\text { trials }\end{array}$ & $\begin{array}{l}\text { False } \\
\text { alarm }\end{array}$ \\
\hline Controls & $3 \cdot 4(2 \cdot 9)$ & $0 \cdot 5(0 \cdot 7)$ & $0 \cdot 4(0 \cdot 6)$ & $0 \cdot 3(0 \cdot 4)$ \\
\hline EOS-Undiff & $3 \cdot 9(2 \cdot 2)$ & $0 \cdot 6(0 \cdot 6)$ & $2 \cdot 4(2 \cdot 8)$ & $0.4(0.8)$ \\
\hline EOS-Paranoid & $4 \cdot 4(3 \cdot 3)$ & $0 \cdot 9(0 \cdot 9)$ & $0 \cdot 3(0 \cdot 5)$ & $0 \cdot 2(0 \cdot 4)$ \\
\hline Overall & $3 \cdot 8(2 \cdot 8)$ & $0 \cdot 7(0 \cdot 7)$ & $0.9(1.8)$ & $0.3(0.6)$ \\
\hline
\end{tabular}

Refer to Table 1 for definitions of error types.

Values are mean (s.D.).

EOS-Undiff, Early-onset schizophrenia undifferentiated; EOSParanoid, early-onset schizophrenia paranoid.

paranoid EOS groups in terms of the SSRT for the left hand $(p>0 \cdot 05)$. The undifferentiated EOS group tended to have longer SSRT with the left hand compared to the right $(p=0.06)$; however, no response hand differences existed for each of the paranoid EOS and control groups. There were no significant differences between the three groups in terms of SSRT for the right hand.

\section{Analysis of Go-RT}

To determine the relationship between mean Go-RT and age we performed partial correlations, adjusting for group. There were significant correlations between age and mean left Go-RT and mean right Go-RT [all $r$ 's $>0.47$ $\left.\left(r^{2}=0 \cdot 22\right)\right]$. Accordingly, we conducted a group by response hand mixed-model ANOVA on mean Go-RT, co-varying for the effects of age. This analysis revealed no main effects of response hand $[F(1,33)=0.259, \quad p=0.614]$ or group [controls: $416 \mathrm{~ms}($ s.D. $=90)$; undifferentiated EOS : 504 ms (s.D. $=106)$; paranoid EOS : $382 \mathrm{~ms}($ S.D. $=47)][F(2,33)=1.748, p=0 \cdot 190]$, nor any interaction between these factors.

\section{Error analyses}

Percentage error rates were calculated for four different error types, including incorrect responses, incorrect failed inhibitions, missed trials and false alarms (see Table 1 for definitions). Percentage error rates as a function of group are presented in Table 2. Preliminary analysis revealed a significant correlation between age and false alarms only (controlling for the effect of Group) $\left(r=-0.43, r^{2}=0.18, p=0.01\right)$. A significant group difference emerged only for missed trials $[F(2,34)=6.632, p=0.004]$. Posthoc analysis with Bonferroni corrections revealed that the undifferentiated EOS group had higher missed trial error rates than either the control $(p<0.05)$ or paranoid EOS group $(p<0.05)$, while the paranoid EOS and control groups did not differ $(p>0 \cdot 05)$. A partial correlation (controlling for group) revealed a significant relationship between percentage missed trials and left-hand SSRT $\left(r=0.45, r^{2}=0 \cdot 20, p=\right.$ $0.006)$ and a trend for a significant correlation between percentage missed trials and right-hand SSRT $\left(r=0 \cdot 31, r^{2}=0 \cdot 09, p=0 \cdot 065\right)$.

\section{DISCUSSION}

This study demonstrates a specific lateralized deficit of response inhibition in adolescents with EOS and high rates of negative symptoms. Specifically, undifferentiated patients had higher negative symptoms than their paranoid counterparts and exhibited prolonged SSRTs when responding with their left, but not right, hand. Furthermore, undifferentiated patients made more errors of omission than either the paranoid patients or the controls, potentially indicating a sustained attention deficit. Importantly, response inhibition deficits in the undifferentiated sample existed in the face of normal response speed, suggesting a dissociation between response inhibition and execution.

Dysfunction to the IFG may be a pathophysiological substrate of negative symptoms in schizophrenia (Wolkin et al. 1992). Levels of negative symptoms may also be higher in patients with relatively earlier illness onsets (Hoff et al. 1996). Kumra et al. (2004) used diffusion tensor imaging to assay white matter integrity in patients with EOS. Relative to control participants, adolescents with EOS had significantly reduced fractional anisotropy in bilateral frontal white matter, particularly in the vicinity of the IFG. Reduced anisotropy within the IFG has also been related to higher levels of impulsivity in adult patients with schizophrenia (Hoptman et al. 2004). These lines of evidence suggest that dysfunction within the right IFG 
may be an attractive neural substrate for the response inhibition deficits observed here in patients with undifferentiated EOS.

Although converging evidence from human lesion and functional neuroimaging studies suggests a crucial role for the right IFG in response inhibition (Garavan et al. 1999; Aron et al. 2003), there is little evidence to suggest that dysfunction within this region can lead to the type of lateralized impairment in response inhibition that we observed in undifferentiated EOS (see Konishi et al. 1998). In fact, using the stop-signal paradigm, we have recently demonstrated that temporary neural disruption caused by TMS of the right IFG impairs response inhibition for both hands in healthy subjects (Chambers et al. in press). We suggest, therefore, that lateralized impairments in response inhibition may arise from dysfunction within motor control areas, such as the SMA and premotor cortex, that have been implicated in both response inhibition (Rubia et al. 2001b; Watanabe et al. 2002) and schizophrenia (Dreher et al. 1999; Payoux et al. 2004; Rogowska et al. 2004). It should be noted that dysfunction to either the left (Flor-Henry, 1976) or right hemisphere (Cutting, 1992) has been suggested in schizophrenia.

Our study is the second to use the stop-signal paradigm in schizophrenia (Badcock et al. 2002) and the first to report an interaction between diagnostic subtype and response hand. Rubia et al. (2001a) used a stop-signal paradigm within a functional imaging design, but their paradigm included only a single stop-signal delay. In their study the stop-signal occurred $250 \mathrm{~ms}$ after the go-stimulus on $30 \%$ of trials. Most previous studies, basic and applied, that have used the stop-signal paradigm have averaged across hand of response and may therefore have overlooked lateralized impairments. Working with an adult cohort of patients with chronic schizophrenia, Badcock et al. (2002) reported normal SSRT relative to psychosis and healthy control groups, but an impaired ability to initiate inhibitory acts. Without including diagnostic subtype as a factor, the current study would also have found no group effect on SSRT. In this respect our study is also compatible with previous reports of unimpaired response inhibition in adult-onset schizophrenia, broadly defined (Fallgatter \&
Muller, 2001; Rubia et al. 2001a). Our results therefore extend this work by demonstrating an interaction between diagnostic subtype and response hand.

The increased proportion of omission errors made by the undifferentiated EOS group is indicative of a sustained attention deficit. Sustained attention deficits are reliably observed in schizophrenia and may be a familial marker of schizophrenia (Cornblatt \& Malhotra, 2001). Imaging studies of sustained attention in drug naïve patients and those experiencing prodromal symptoms of the disorder show functional deficits in the inferior frontal gyrus (Ojeda et al. 2002; Morey et al. 2005). The overlapping neuroanatomical substrates of sustained attention and response inhibition may suggest a functional link between these processes.

Maturational delays in the development of the brain are thought to confer susceptibility to an early illness onset in adolescents with schizophrenia. Supporting evidence for this hypothesis comes from studies reporting delays in cognitive, linguistic and social development (Hollis, 1995) and the persistence of neurological soft signs at a developmentally inappropriate age (Karp et al. 2001). Neuroimaging has begun to provide preliminary evidence for this hypothesis, with pronounced structural changes in the frontal lobe of EOS that appear larger than those in comparable studies of adult-onset patients (James et al. 2004). In addition, however, comparable changes in frontal microstructure have been reported across early-onset (Kumra et al. 2004) and adult-onset samples (Buchsbaum et al. 1998; Lim et al. 1999). The application of neurocognitive measures with known brain-behaviour relationships, such as response inhibition, may provide an additional and important source of information. In this context, the observation of a lateralized impairment in response inhibition in EOS is an important finding. Promising candidates for future functional imaging studies may be the roles of the IFG, SMA and pre-motor cortex in response inhibition, particularly in earlyonset patients with high levels of negative symptomatology.

Given the low prevalence of EOS, this study was probably underpowered. The difference between the SSRTs, irrespective of hand of response, of the undifferentiated EOS group 
and both the paranoid EOS and control groups were of large effect size (Cohen's $d=0.74$ and 0.72 respectively). This suggests that larger sample sizes are likely to reveal both main effects of diagnostic group on SSRT and an interaction between diagnostic group and hand of response. These results would then be indicative of a broad disruption to inhibitory networks in undifferentiated EOS, including the right IFG and associated regions such as the pre-motor cortex/SMA that are thought to play a role in both motoric initiation and inhibition.

In summary, we have identified a specific lateralized deficit of response inhibition in EOS. As we did not observe any group differences for response speed, our results are unlikely to reflect a generalized cognitive impairment. Instead, our findings add to a developing literature on neurocognitive deficits in EOS (Bellgrove et al. 2003, 2004; Kravariti et al. 2003 a; McClellan et al. 2004; Tuulio-Henriksson et al. 2004). An important goal for future studies will be to determine whether response inhibition deficits may serve as a marker for vulnerability to an early onset of schizophrenia.

\section{ACKNOWLEDGEMENTS}

This work was, in part, carried out as part of the doctoral thesis of M.A.B. at Monash University, Australia. Subsequently, this work was supported by an Australian Rotary Health Research Fund to M.A.B, A.V. and J.L.B. M.A.B is currently supported by a National Health and Medical Research Council (Australia) Howard Florey Centenary Fellowship.

\section{DECLARATION OF INTEREST}

None.

\section{REFERENCES}

APA (1994). Diagnostic and Statistical Manual of Mental Disorders : DSM-IV (4th edn). Washington, DC: American Psychiatric Association.

Aron, A. R., Fletcher, P., Bullmore, E. T., Sahakian, B. J. \& Robbins, T. W. (2003). Stop-signal inhibition disrupted by damage to the right inferior frontal gyrus in humans. Nature Neuroscience 6, $115-116$.

Badcock, J. C., Michie, P. T., Johnson, L. \& Combrinck, J. (2002). Acts of control in schizophrenia: dissociating the components of inhibition. Psychological Medicine 32, 287-297.
Bellgrove, M. A., Collinson, S., Mattingley, J. B., Pantelis, C., Fitzgerald, P. B., James, A. C. \& Bradshaw, J. L. (2004). Attenuation of perceptual asymmetries in patients with early-onset schizophrenia: evidence in favour of reduced hemispheric differentiation in schizophrenia. Laterality 9, 79-91.

Bellgrove, M. A., Vance, A. \& Bradshaw, J. L. (2003). Local-global processing in early-onset schizophrenia: evidence for an impairment in shifting the spatial scale of attention. Brain and Cognition 51, 48-65.

Berman, K. F., Zec, R. F. \& Weinberger, D. R. (1986). Physiologic dysfunction of dorsolateral prefrontal cortex in schizophrenia. II Role of neuroleptic treatment, attention, and mental effort. Archives of General Psychiatry 43, 126-135.

Bleuler, E. (1911). Dementia Praecox or the Group of Schizophrenias (translated by J. Zinkin, 1950). International Universities Press: New York.

Buchsbaum, M. S., Haier, R. J., Potkin, S. G., Nuechterlein, K., Bracha, H. S., Katz, M., Lohr, J., Wu, J., Lottendberg, S., Jerabek, P. A., Trenary, M., Tafalla, R., Reynolds, C. \& Bunney, W. E. (1992). Frontostriatal disorder of cerebral metabolism in nevermedicated schizophrenics. Archives of General Psychiatry 49, 935-942.

Buchsbaum, M. S., Tang, C. Y., Peled, S., Gudbjartsson, H., Lu, D., Hazlett, E. A., Downhill, J., Haznedar, M., Fallon, J. H. \& Atlas, S. W. (1998). MRI white matter diffusion anisotropy and PET metabolic rate in schizophrenia. Neuroreport 9, 425-430.

Carter, C. S., Braver, T. S., Barch, D. M., Botvinick, M. M., Noll, D. \& Cohen, J. D. (1998). Anterior cingulate cortex, error detection, and the online monitoring of performance. Science 280, 747-749.

Chambers, C. D., Bellgrove, M. A., Stokes, M. G., Henderson, T. R., Garavan, H., Robertson, I. H., Morris, A. P. \& Mattingley, J. B. (in press). Executive 'brake failure' following deactivation of human frontal lobe. Journal of Cognitive Neuroscience.

Cornblatt, B. A. \& Malhotra, A. K. (2001). Impaired attention as an endophenotype for molecular genetic studies of schizophrenia. American Journal of Medical Genetics 105, 11-15.

Cutting, J. (1992). The role of right hemisphere dysfunction in psychiatric disorders. British Journal of Psychiatry 160, 583-588.

Dreher, J. C., Trapp, W., Banquet, J. P., Keil, M., Gunther, W. \& Burnod, Y. (1999). Planning dysfunction in schizophrenia: impairment of potentials preceding fixed/free and single/sequence of self-initiated finger movements. Experimental Brain Research 124, 200-214.

Fallgatter, A. J. \& Muller, T. J. (2001). Electrophysiological signs of reduced prefrontal response control in schizophrenic patients. Psychiatry Research 107, 19-28.

Flor-Henry, P. (1976). Lateralized temporo-limbic dysfunction and psychopathology. Annals of the New York Academy of Science $\mathbf{2 8 0}$ 777-795.

Ford, J. M., Gray, M., Whitfield, S. L., Turken, A. U., Glover, G., Faustman, W. O. \& Mathalon, D. H. (2004). Acquiring and inhibiting prepotent responses in schizophrenia: event-related brain potentials and functional magnetic resonance imaging. Archives of General Psychiatry 61, 119-129.

Funnell, M., Gazzaniga, M. \& Garavan, H. (2004). Cognitive Neuroscience Society Annual Meeting, San Francisco [Abstract].

Garavan, H., Ross, T. J., Kaufman, J. \& Stein, E. A. (2003). A midline dissociation between error-processing and response-conflict monitoring. Neuroimage 20, 1132-1139.

Garavan, H., Ross, T. J. \& Stein, E. A. (1999). Right hemisphere dominance for inhibitory control: an event-related functional MRI study. Proceedings of the National Academy of Sciences of the United States of America 96, 8301-8306.

Hill, K., Mann, L., Laws, K. R., Stephenson, C. M., Nimmo-Smith, I. \& McKenna, P. J. (2004). Hypofrontality in schizophrenia: a meta-analysis of functional imaging studies. Acta Psychiatrica Scandinavica 110, 243-256.

Hoff, A. L., Harris, D., Faustman, W. O., Beal, M., DeVilliers, D., Mone, R. D., Moses, J. A. \& Csernansky, J. G. (1996). A neuropsychological study of early onset schizophrenia. Schizophrenia Research 20, 21-28. 
Hollis, C. (1995). Child and adolescent (juvenile onset) schizophrenia. A case control study of premorbid developmental impairments. British Journal of Psychiatry 166, 489-495.

Hollis, C. (2000). Adult outcomes of child- and adolescentonset schizophrenia: diagnostic stability and predictive validity. American Journal of Psychiatry 157, 1652-1659.

Hoptman, M. J., Ardekani, B. A., Butler, P. D., Nierenberg, J., Javitt, D. C. \& Lim, K. O. (2004). DTI and impulsivity in schizophrenia: a first voxelwise correlational analysis. Neuroreport 15, 2467-2470

James, A. C., James, S., Smith, D. M. \& Javaloyes, A. (2004) Cerebellar, prefrontal cortex, and thalamic volumes over two time points in adolescent-onset schizophrenia. American Journal of Psychiatry 161, 1023-1029.

Karp, B. I., Garvey, M., Jacobsen, L. K., Frazier, J. A., Hamburger, S. D., Bedwell, J. S. \& Rapoport, J. L. (2001). Abnormal neurologic maturation in adolescents with early-onset schizophrenia. American Journal of Psychiatry 158, 118-122.

Kaufman, J., Birmaher, B., Brent, D., Rao, U., Flynn, C., Moreci, P., Williamson, D. \& Ryan, N. (1999). Schedule for Affective Disorders and Schizophrenia for School-Age Children - Present and Lifetime Version (K-SADS-PL): initial reliability and validity data. Journal of the American Academy of Child and Adolescent Psychiatry 36, 980-988.

Kay, S. R., Fiszbein, A. \& Opler, L. A. (1987). The Positive and Negative Syndrome Scale (PANSS) for schizophrenia. Schizophrenia Bulletin 13, 261-276.

Kelly, A. M., Hester, R., Murphy, K., Javitt, D. C., Foxe, J. J. \& Garavan, H. (2004). Prefrontal-subcortical dissociations underlying inhibitory control revealed by event-related fMRI. European Journal of Neuroscience 19, 3105-3112.

Kiehl, K. A., Smith, A. M., Hare, R. D. \& Liddle, P. F. (2000) An event-related potential investigation of response inhibition in schizophrenia and psychopathy. Biological Psychiatry 48, 210 221

Kolb, B. \& Wishaw, I. Q. (1983). Performance of schizophrenic patients on tests sensitive to left or right frontal temporal, or parietal function in neurological patients. Journal of Nervous and Mental Disorders 171, 435-443.

Konishi, S., Nakajima, K., Uchida, I., Sekihara, K. \& Miyashita, Y. (1998). No-go dominant brain activity in human inferior prefrontal cortex revealed by functional magnetic resonance imaging. European Journal of Neuroscience 10, 1209-1213.

Kornblum, S., Hasbroucq, T. \& Osman, A. (1990). Dimensional overlap: cognitive basis for stimulus-response compatibility - a model and taxonomy. Psychological Review 97, 253-270.

Kraepelin, E. (1913). Dementia Praecox and Paraphrenia (translated by R. M. Barclay, 1919). E. \& S. Livingstone: Edinburgh.

Kravariti, E., Morris, R. G., Rabe-Hesketh, S., Murray, R. M. \& Frangou, S. (2003a). The Maudsley Early-Onset Schizophrenia Study: cognitive function in adolescents with recent onset schizophrenia. Schizophrenia Research 61, 137-148.

Kravariti, E., Morris, R. G., Rabe-Hesketh, S., Murray, R. M. \& Frangou, S. $(2003 b)$. The Maudsley Early-Onset Schizophrenia Study: cognitive function in adolescent-onset schizophrenia. Schizophrenia Research 65, 95-103.

Kumra, S., Ashtari, M., McMeniman, M., Vogel, J., Augustin, R., Becker, D. E., Nakayama, E., Gyato, K., Kane, J. M., Lim, K. \& Szeszko, P. (2004). Reduced frontal white matter integrity in early-onset schizophrenia: a preliminary study. Biological Psychiatry 55, 1138-1145.

Levitt, H. (1971). Transformed up-down methods in psychoacoustics. Journal of the Acoustical Society of America 49, 467-477.

Lim, K. O., Hedehus, M., Moseley, M., de Crespigny, A., Sullivan, E. V. \& Pfefferbaum, A. (1999). Compromised white matter tract integrity in schizophrenia inferred from diffusion tensor imaging. Archives of General Psychiatry 56, 367-374.

Logan, G. D. (1994). On the ability to inhibit thought and action: a user's guide to the Stop Signal Paradigm. In Inhibitory Processes in Attention, Memory, and Language (ed. D. Dagenbach and T. H. Carr), pp. 189-239. Academic Press: San Diego.
Logan, G. D. \& Cowan, W. B. (1984). On the ability to inhibit thought and action: a theory of an act of control. Psychological Review 91, 295-327.

Logan, G. D., Schachar, R. J. \& Tannock, R. (1997). Impulsivity and inhibitory control. Psychological Science 8, 60-64.

McClellan, J., Prezbindowski, A., Breiger, D. \& McCurry, C. (2004). Neuropsychological functioning in early onset psychotic disorders. Schizophrenia Research 68, 21-26.

Miyake, A., Friedman, N. P., Emerson, M. J., Witzki, A. H., Howerter, A. \& Wager, T. D. (2000). The unity and diversity of executive functions and their contributions to complex 'frontal lobe' tasks: a latent variable analysis. Cognitive Psychology 41, 49-100.

Morey, R. A., Inan, S., Mitchell, T. V., Perkins, D. O., Lieberman, J. A. \& Belger, A. (2005). Imaging frontostriatal function in ultrahigh-risk, early, and chronic schizophrenia during executive processing. Archives of General Psychiatry 62, 254-262.

Nicolson, R., Lenane, M., Hamburger, S. D., Fernandez, T., Bedwell, J. \& Rapoport, J. L. (2000). Lessons from childhood-onset schizophrenia. Brain Research Brain. Research Reviews 31, 147-156.

Ojeda, N., Ortuno, F., Arbizu, J., Lopez, P., Marti-Climent, J. M., Penuelas, I. \& Cervera-Enguix, S. (2002). Functional neuroanatomy of sustained attention in schizophrenia: contribution of parietal cortices. Human Brain Mapping 17, 116-130.

Osman, A., Kornblum, S. \& Meyer, D. E. (1986). The point of no return in choice reaction time: controlled and ballistic stages of response preparation. Journal of Experimental Psychology: Human Perception and Performance 12, 243-258.

Osman, A., Kornblum, S. \& Meyer, D. E. (1990). Does motor programming necessitate response execution. Journal of Experimental Psychology: Human, Perception and Performance 16, 183-198.

Pantelis, C., Barnes, T. R. E., Nelson, H. E., Tanner, S., Weatherley, L., Owen, A. M. \& Robbins, T. W. (1997). Frontal-striatal cognitive deficits in patients with chronic schizophrenia. Brain 120, 101-120.

Pantelis, C., Yucel, M., Wood, S. J., McGorry, P. D. \& Velakoulis, D. (2003). Early and late neurodevelopmental disturbances in schizophrenia and their functional consequences. Australian and New Zealand Journal of Psychiatry 37, 399-406.

Patterson, K. \& Bradshaw, J. L. (1975). Differential hemispheric mediation of nonverbal visual stimuli. Journal of Experimental Psychology: Human Perception and Performance 1, 246-252.

Payoux, P., Boulanouar, K., Sarramon, C., Fabre, N., Descombes, S., Galitsky, M., Thalamas, C., Brefel-Courbon, C., Sabatini, U., Manelfe, C., Chollet, F., Schmitt, L. \& Rascol, O. (2004). Cortical motor activation in akinetic schizophrenic patients: a pilot functional MRI study. Movement Disorders 19, 83-90.

Pennington, B. F. \& Ozonoff, S. (1996). Executive functions and developmental psychopathology. Journal of Child Psychology and Psychiatry 37, 51-87.

Rogowska, J., Gruber, S. A. \& Yurgelun-Todd, D. A. (2004) Functional magnetic resonance imaging in schizophrenia: cortical response to motor stimulation. Psychiatry Research 130, 227-243.

Rubia, K., Russell, T., Bullmore, E. T., Soni, W., Brammer, M. J., Simmons, A., Taylor, E., Andrew, C., Giampietro, V. \& Sharma, T. $(2001 a)$. An fMRI study of reduced left prefrontal activation in schizophrenia during normal inhibitory function. Schizophrenia Research 52, 47-55.

Rubia, K., Russell, T., Overmeyer, S., Brammer, M. J., Bullmore, E. T., Sharma, T., Simmons, A., Williams, S. C., Giampietro, V., Andrew, C. M. \& Taylor, E. (2001 b). Mapping motor inhibition: conjunctive brain activations across different versions of go/no-go and stop tasks. Neuroimage 13, 250-261.

Tuulio-Henriksson, A., Partonen, T., Suvisaari, J., Haukka, J. \& Lonnqvist, J. (2004). Age at onset and cognitive functioning in schizophrenia. British Journal of Psychiatry 185, 215-219.

Watanabe, J., Sugiura, M., Sato, K., Sato, Y., Maeda, Y., Matsue, Y., Fukuda, H. \& Kawashima, R. (2002). The human prefrontal and parietal association cortices are involved in no-go performances: an event-related fMRI study. Neuroimage 17, 1207-1216. 
Weisbrod, M., Kiefer, M., Marzinzik, F. \& Spitzer, M. (2000). Executive control is disturbed in schizophrenia: evidence from event-related potentials in a Go/NoGo task. Biological Psychiatry 47, 51-60.

Wolkin, A., Choi, S. J., Szilagyi, S., Sanfilipo, M., Rotrosen, J. P. \& Lim, K. O. (2003). Inferior frontal white matter anisotropy and negative symptoms of schizophrenia: a diffusion tensor imaging study. American Journal of Psychiatry 160, 572-574.

Wolkin, A., Sanfilip, M., Wolf, A. P., Angrist, B., Brodie, J. D. \&

Rotrosen, J. (1992). Negative symptoms and hypofrontality in chronic schizophrenia. Archives of General Psychiatry 49, 959-965. 